\title{
RELATIONS BETWEEN MOVEMENT AND SINGLE CELL DISCHARGE IN THE SUBSTANTIA NIGRA OF THE BEHAVING MONKEY ${ }^{1}$
}

\author{
MAHLON R. DeLONG, ${ }^{2}$ MICHAEL D. CRUTCHER, ${ }^{3}$ AND APOSTOLOS P. GEORGOPOULOS \\ Departments of Neuroscience, Neurology, and Physiology, The Johns Hopkins University School of Medicine, \\ Baltimore, Maryland 21205
}

Rcceived July 20, 1982; Revised February 22, 1983; Accepted March 7, 1983

\begin{abstract}
In order to clarify the motor functions of the substantia nigra (SN), we studied the activity of single neurons in both the pars compacta $(\mathrm{SNpc})$ and the pars reticulata (SNpr) of behaving primates during performance of a visuomotor arm-tracking task. We also studied the relations of neuronal activity to active movements and passive manipulations of the limbs and other body parts outside the behavioral paradigm.

On the basis of spontaneous discharge rates, most cells within the $\mathrm{SN}$ fell into two categories: (1) low discharge rate (LDR) cells $(n=53$, mean rate 2.1 impulses $/ \mathrm{sec})$ and $(2)$ high discharge rate (HDR) cells $(n=129$, mean rate 60.4 impulses/sec). HDR neurons had firing rates and discharge patterns similar to neurons in the inner segment of the globus pallidus. Most (83\%) LDR neurons were located within the SNpc, and all HDR neurons were located in the SNpr.

None of the LDR neurons in SNpc exhibited detectable phasic changes in discharge in relation to active movements or passive manipulations. Only a small number of SNpc cells showed modulation in the behavioral paradigm. These findings suggest that the nigrostriatal dopamine system, rather than conveying highly specific information about movement to the striatum, may exert a more tonic modulatory effect upon the striatum. However, phasic release of dopamine in the striatum may be affected by presynaptic mechanisms.

Twenty-five percent of cells in the SNpr were related to licking and chewing movements. Cells specifically related to limb movements were rare. Some of these arm movement-related neurons showed a relation to movement parameters. A small number of SNpr cells $(3 \%)$ were related to eye movements or exhibited responses to gross visual stimulation. Neurons related to licking and chewing were located primarily in the centrolateral portions of the nucleus. These findings suggest that the lateral portion of the SNpr may play a role in the control of orofacial movements.
\end{abstract}

Abundant clinicopathological and experimental evidence indicates an important role of the substantia nigra in motor function (Jung and Hassler, 1960; Martin, 1967; DeLong and Georgopoulos, 1981). In Parkinson's disease loss of the dopamine-containing cells in the pars compacta of the substantia nigra ( $\mathrm{SNpc}$ ) is associated with severe motor abnormalities, including akinesia and rigidity. In the rat, interruption of the ascending dopamine (DA) projections results in profound abnormalities in posture and movement (Ungerstedt, 1971). In primates,

\footnotetext{
${ }^{1}$ This work supported by United States Public Health Service Grants NS 15417, NS 06828, and NS 07226.

${ }^{2}$ To whom correspondence should be addressed at Department of Neurology, 122 B Building, Baltimore City Hospitals, 4940 Eastern Avenue, Baltimore, MD 21224.

${ }^{3}$ Present address: Division of Neurobiology and Behavior, Columbia University, CPS, 630 W. 168th Street, New York, NY 10032.
}

coagulative lesions of the substantia nigra (SN) result in hypokinesia (Stern, 1966; Viallet et al., 1981). It is clear that the normal functioning of the basal ganglia depends upon an intact nigrostriatal DA system. Nigrostriatal DA neurons could play a role in motor control in several ways: they could, for example, encode in their discharge information about specific parameters (e.g., direction, amplitude, or velocity) of movement or, alternatively, could have a general modulatory effect upon the striatum, where detailed aspects of movement could be controlled.

Little is known of the role of the SNpr, although the afferents to this portion of the SN from the putamen (Szabo, 1967) and the efferent projections to portions of the ventrolateral and ventroanterior nuclei of the thalamus (Carpenter et al., 1976) suggest a role in motor function. The effects of coagulative lesions of the $\mathrm{SN}$ on 
motor function are generally attributed to damage to the SNpc. However, such lesions invariably include portions of the SNpr, which gives rise to major projections from the basal ganglia to the thalamus, superior colliculus, and midbrain (Mehler, 1971; Carpenter et al., 1976; Graybiel, 1978).

In order to clarify the functions of the SNpc and SNpr, we studied the activity of single neurons in these nuclei in the primate during performance of a visuornotor armtracking task, and in relation to active movements and passive manipulations of the limbs and other body parts outside the behavioral paradigm. By the latter approach, we sought to characterize the functional properties of SN neurons in relation to a wider range of movements and in response to natural somatosensory stimuli. These studies in the SN were an extension of similar studies in the globus pallidus and subthalamic nucleus (Georgopoulos et al., 1983).

Some of the findings have been presented in preliminary form (DeLong and Georgopoulos, 1978, 1979, 1981).

\section{Materials and Methods}

Three rhesus monkeys weighing between 4 and $5 \mathrm{~kg}$ were used in the present experiments. These were the same animals for which the relations to movement in the globus pallidus and subthalamic nucleus are described in the companion paper (Georgopoulos et al., 1983). The methods of animal training and examination, histological reconstruction, extracellular recording and data analysis, and the details of the behavioral paradigm have been described fully in the companion paper (Georgopoulos et al., 1983). Briefly, the animals were trained to allow passive manipulations of the limbs and trunk by offering food rewards. Responses to joint rotation, muscle and tendon taps, light touch of the skin and hair were determined; also, changes in neuronal discharge during active movements of the animal's limbs, face, trunk, and eyes were observed. On the basis of correlations between neuronal discharge and active movements and/or passive manipulations of specific body parts, cells were categorized, when possible, as related to arm, leg, orofacial $(\mathrm{OF})$, trunk, or eye movements. Cells showing a definite alteration of discharge during the examination of the animal, but without a clearly discernible relation to active movements or stimulation of specific body parts, were categorized as nonspecific (NS). Cells showing no discernible alteration of discharge during examination were termed nonresponsive (NR).

Animals were trained to perform a visuomotor tracking task in which the animal grasped and moved a lightweight, low-friction handle from side to side or in a pushpull direction. Animals observed a display which consisted of two rows of light-emitting diodes (LEDs) arranged in two horizontal rows, one below the other. Each row contained $128 \mathrm{LEDs}$ and was $32 \mathrm{~cm}$ long. The illuminated LED of the upper row indicated the target position; the illuminated LED of the lower row corresponded to the current position of the handle. The illumination was enhanced when the two LEDs were aligned within a positional window. The animal was required to move the handle so as to align the lower LED with the upper LED. A trial began by turning on the target LED at an initial (starting) position. The animal had to move the manipulandum to align the handle position LED with that of the target LED and hold it in that position for at least 2 sec. The initial LED was then turned off, a new (target) LED was lighted, and the animal had to move the handle and align its LED below the target LED. A liquid reward was delivered after the animal held the handle for at least $0.5 \mathrm{sec}$ at the target position.

Both the direction and the amplitude of movement were varied in the task. The effects of direction and amplitude of movements on the neuronal discharge rate were evaluated using standard statistical techniques (see companion paper, Georgopoulos et al., 1983).

We sought to sample the $\mathrm{SN}$ as uniformly as possible with penetrations separated by $1 \mathrm{~mm}$. Records were kept of the depth of each cell isolated along each penetration, from the earliest activity in the cortex to the termination of the penetration below the SN. Electrolytic marking lesions were made after completion of the recording period to verify recording sites. In addition to marking lesions, the patterns of the neural activity in different regions were helpful in identifying the location of neurons both during the recording sessions as well as in subsequent reconstruction of the penetrations. During rostral and lateral penetrations, entry into the SNpr was preceded by passage of the microelectrode through the subthalamic nucleus with its characteristic spontaneous activity (Georgopoulos et al., 1983). Entry into the SN in these lateral penetrations was reliably signaled by the appearance of neurons with high frequency discharge characteristic of the SNpr. Entry of the recording electrode into the medial and caudal portion of the $\mathrm{SN}$ where the pars compacta is present was less clearly determined at the time of recording because of the very low discharge rates of neurons in this region and the lack of a clear change from cell activity in the overlying region.

\section{Results}

The activity of 190 neurons in the SN was studied in 45 histologically identified penetrations in four hemispheres of three animals. Figure 1 shows a representative coronal section from one animal with gliotic tracks from electrode penetrations and marking lesions in the SN.

\section{Spontaneous activity}

Cells within the SN could be grouped into two types on the basis of their spontaneous discharge rates: (1) low discharge rate (LDR, $n=53$ ) and (2) high discharge rate (HDR, $n=129$ ) cells. The mean discharge rate of LDR cells was 2.1 impluses/sec (range from 0.2 to 6.0 impulses/sec), whereas the mean discharge rate of HDR cells was $60.4 \mathrm{impulses} / \mathrm{sec}$ (range from 33 to 114 impulses/ sec.). A small number of cells $(n=8)$ had discharge rates between 10 and 30 impulses/sec.

\section{Location of cell types}

Most (44 of 53) LDR neurons were located within the SNpc, and all HDR neurons were found in the SNpr. This is seen in Figure 2, which shows the locations of LDR and HDR cells within the SN in one animal. The preferential localization of HDR cells within the SNpr was most apparent in rostral and lateral portions of the 

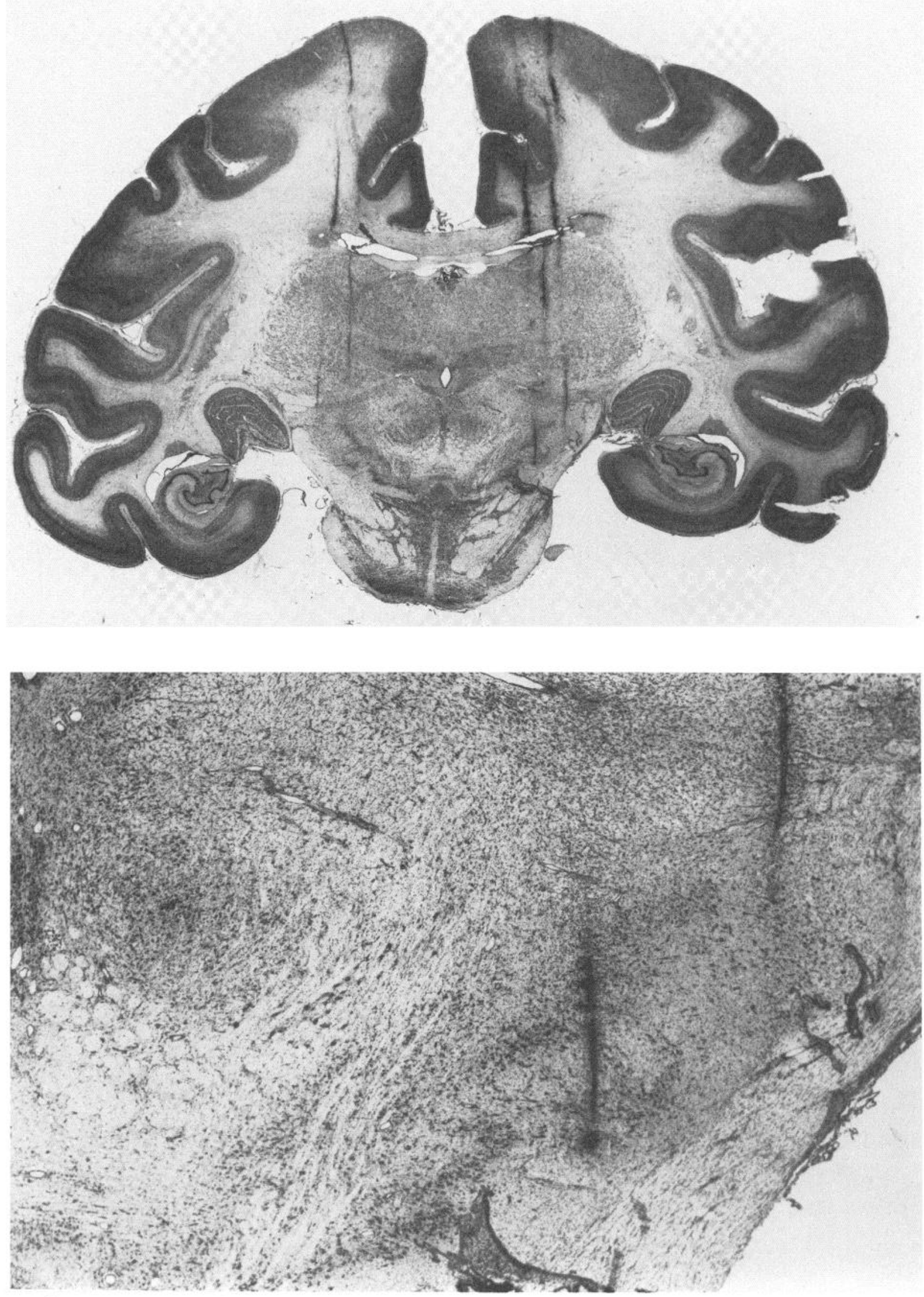

Figure 1. Coronal section through the $\mathrm{SN}$ showing representative electrode penetrations through the $\mathrm{SN}$ of both hemispheres (above). The enlargement of the region of the $\mathrm{SN}$ of the right hemisphere (below) shows a typical marking lesion within the $\mathrm{SN}$. Lesions were made by passing current $(10 \mu \mathrm{A} \times 10 \mathrm{sec})$ through the recording microelectrode. 


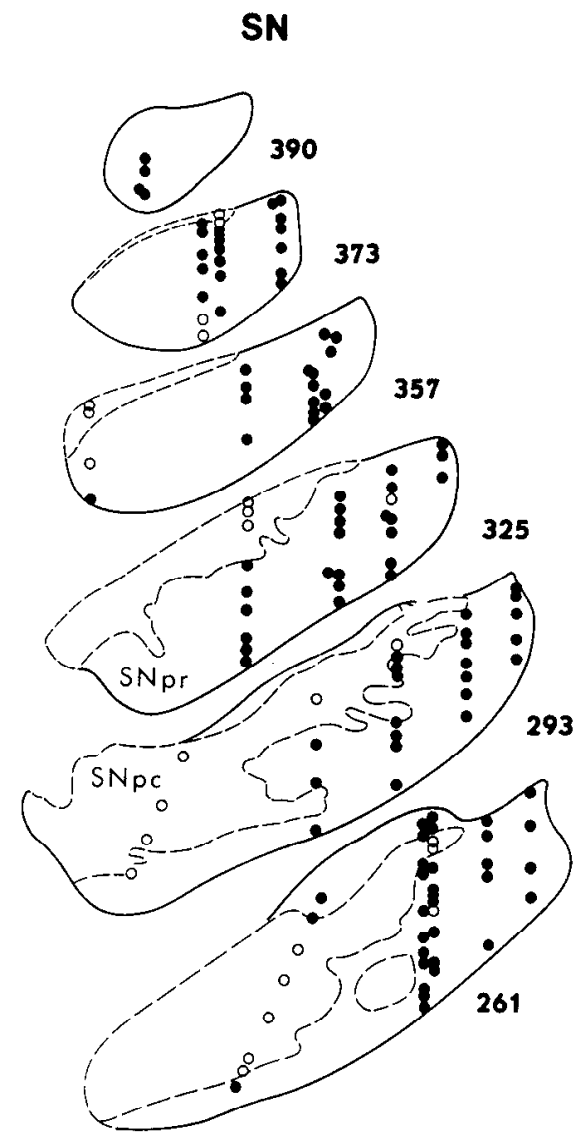

Figure 2. Location of LDR (open circles) and HDR (solid circles) neurons from two animals. The numbers represent sections. The brain was sectioned at $25-\mu$ intervals. Higher numbers represent more rostral sections. The $\mathrm{SNpc}$ is the portion of SN delimited by the dotted lines.

nucleus where the $\mathrm{SNpc}$ is absent. Conversely, the preferential localization of LDR neurons in the SNpc was best seen in the caudal and medial portions where the $\mathrm{SNpc}$ is most developed. The localization of several cells $(n=8)$ situated at the junction between the SNpc and SNpr could not be positively determined because of the irregular and sometimes uncertain border between these two regions.

\section{Relations to movement}

$S N p c$

Of 44 LDR neurons located within SNpc, only $2(4 \%)$ exhibited any discernible change in discharge during the examination (Table I). None of the SNpc cells were phasically related to movements of individual body parts. When tested in the behavioral paradigm, however, a small number of SNpc cells $(n=3)$ showed weak modulation. One exhibited a general increase in discharge during the entire period of task performance and became silent when the task ended. Another showed a weak decrease in discharge during the reaction time period of the task. The third, shown in Figure 3, showed a sustained inhibition following the stimulus presentation in all trials which lasted to the end of each trial. No significant relations to specific movement parameters (direc-
TABLE I

Classification of cells in SNpc and SNpr based on responses to active movements and passive manipulations

\begin{tabular}{lrrrrr}
\hline & \multicolumn{2}{c}{ SNpr } & & \multicolumn{2}{c}{ SNpc } \\
\cline { 2 - 3 } \cline { 5 - 6 } & No. & $\%$ & & No. & $\%$ \\
\hline Arm & 7 & 5 & & 0 & 0 \\
Leg & 1 & 1 & & 0 & 0 \\
Orofacial & 34 & 25 & & 0 & 0 \\
Axial & 2 & 1 & & 0 & 0 \\
Eye movement & 4 & 3 & & 0 & 0 \\
Nonspecific & 20 & 14 & & 2 & 4 \\
Nonresponsive & 70 & 51 & & $\frac{42}{44}$ & $\frac{96}{100}$ \\
\hline
\end{tabular}

tion, velocity, or amplitude) were observed in $\mathrm{SNpc}$ neurons.

SNpr

As shown in Table I, $25 \%$ of all SNpr cells were related to licking and/or chewing movements (OF cells). Nearly all OF cells were related to licking and/or chewing movements both during and outside the behavioral paradigm. None of the OF cells appeared to be related to movements of the face (i.e., lips, eyelids, brow), per se. Neurons related to limb movements were infrequent; seven cells were related to reaching movements of the arm and one to leg movements. None of the arm cells were related to distal movements of the limbs. An example of one HDR arm neuron which was related to the step-tracking task is shown in Figure 4. The activity of this cell exhibited a relation to both the direction (not shown in Fig. 4) and the amplitude of movement. Two of the seven arm cells showed a relation to the amplitude of movement.

A small number of cells (3\%) were related to spontaneous saccadic eye movements or exhibited responses to gross visual stimulation. Cells related to eye movements typically showed an inhibition of discharge during the eye movements as described by Hikosaka and Wurtz (1981).

Whereas only a very small percentage of LDR cells located within the SNpc showed increases in discharge during the examination, it is noteworthy that eight of the nine LDR cells in the SNpr did so. Six of these showed nonspecific changes which appeared to be largely related to the animal's level of arousal, and two showed changes related to orofacial movements.

\section{Responses to passive manipulations}

Responses to passive manipulations of the limbs were rare, and no responses to manipulations of the distal parts of the limb were seen. Some of the cells related to licking and chewing also responded to sensory stimulation of the tongue or jaw.

\section{Functional grouping}

Neurons related to licking and chewing (OF cells) were located primarily in the centrolateral portions of the nucleus, as shown in Figures 5 and 6. Typically, several $\mathrm{OF}$ cells were encountered along a given penetration in this region. Five of the seven arm-related cells were found in the caudal part of the SNpr. 


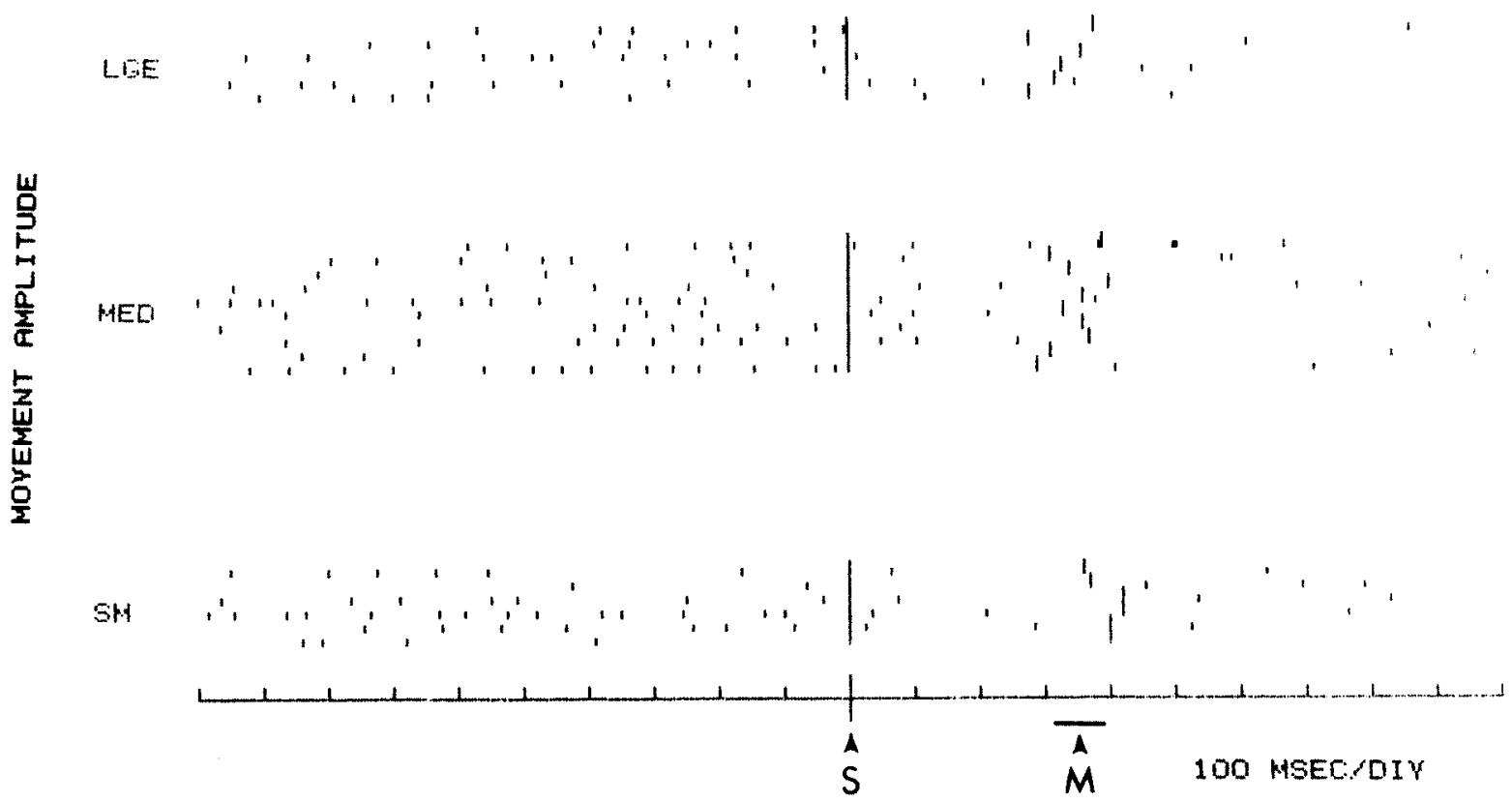

Figure 3. Stimulus-aligned raster of the activity of an $\mathrm{SNpc}$ neuron in the behavioral paradigm. $S M, M E D$, and $L G E$ represent step movements of $20 \mathrm{~mm}, 62.5 \mathrm{~mm}$, and $100 \mathrm{~mm}$, respectively. $S$, appearance of the target stimulus; $M$, onset of movement ( \pm 1 $\mathrm{SD})$. Vertical marks indicate the time of onset of movement for each trial.

\section{Discussion}

$S N p c$

The finding that neurons in the SNpc exhibit low discharge rates whereas neurons in the SNpr exhibit high discharge rates is consistent with the results of earlier studies in the rat (Bunney et al., 1973; Guyenet and Aghajanian, 1978) and monkey (Anderson, 1976). Our findings, however, only partially agree with those of Feger et al. (1978), who found no cells in the SN in the monkey with rates below $10 / \mathrm{sec}$.

In the rat, antidromic stimulation studies indicate that low discharge rate "type I" cells in the SNpc project to the striatum, whereas most high discharge rate "type II" cells in the SNpr project to the thalamus (Guyenet and Aghajanian, 1978). In addition, type I cells were inhibited by the administration of apomorphine or the iontophoretic application of DA or GABA, whereas type II cells were inhibited by the iontophoretic application of GABA but not DA. Although such pharmacological manipulations were not carried out in our studies, it is probable that the majority of LDR neurons located in the SNpc in this study, as in the rat, project to the striatum and that most HDR cells in the SNpr give rise to the extrinsic projections of the SNpr to the thalamus, superior colliculus, and midbrain.

It is of some interest that, in spite of the far greater density of cells in the SNpc than in the SNpr, the number of cells recorded per millimeter of travel of the electrode was no greater than in the SNpr (e.g., see Fig. 2). This, together with the very low rates of spontaneous discharge of most recorded SNpc cells, suggests that many of the neurons in the pars compacta in the awake monkey are not spontaneously active or fire very infrequently.

None of the SNpc neurons exhibited phasic changes in firing in relation to active movements or passive manipulations outside of the task. Even the three SNpc cells which exhibited a change in discharge during movements in the task did not appear to encode information about movement parameters (direction, amplitude, or velocity). The lack of modulation of $\mathrm{SNpc}$ neurons during movement in the intact animal has been recently confirmed in the freely moving cat (Steinfels et al., 1981). The present findings are in striking contrast to the results of similar studies in behaving primates in the globus pallidus, subthalamic nucleus (Georgopoulos et al., 1983), and putamen (Crutcher and DeLong, 1981, 1983), which have revealed specific relations between cell discharge and both movement of individual body parts and parameters of movement.

The lack of observed modulation of SNpc neurons by somatosensory stimulation in these studies is difficult to reconcile with the reports of such modulation in the anesthetized rat (Hommer and Bunney, 1980) and monkey (Feger et al., 1978). This discrepancy may be due to the use of anesthetics in the other studies or to the fact that the level of neuronal activity in the SNpc in these studies appears to have been greater, thus allowing inhibitory effects to be seen more readily.

The present findings support the view that the nigrostriatal DA system, rather than conveying specific information about movement, may exert for the most part a more "tonic," modulatory action upon the striatum. Several independent lines of evidence are consistent with this view: (I) the relatively small absolute number of nigrostriatal DA neurons and their rather divergent projections to the striatum (Fallon and Moore, 1978); (2) the slow conduction velocities of their axons (Guyenet and Aghajanian, 1978); (3) the beneficial effects of Ldopa and direct-acting dopamine receptor agonists (such as apomorphine) on the behavioral disturbances resulting from lesions of the nigrostriatal DA pathways; and 

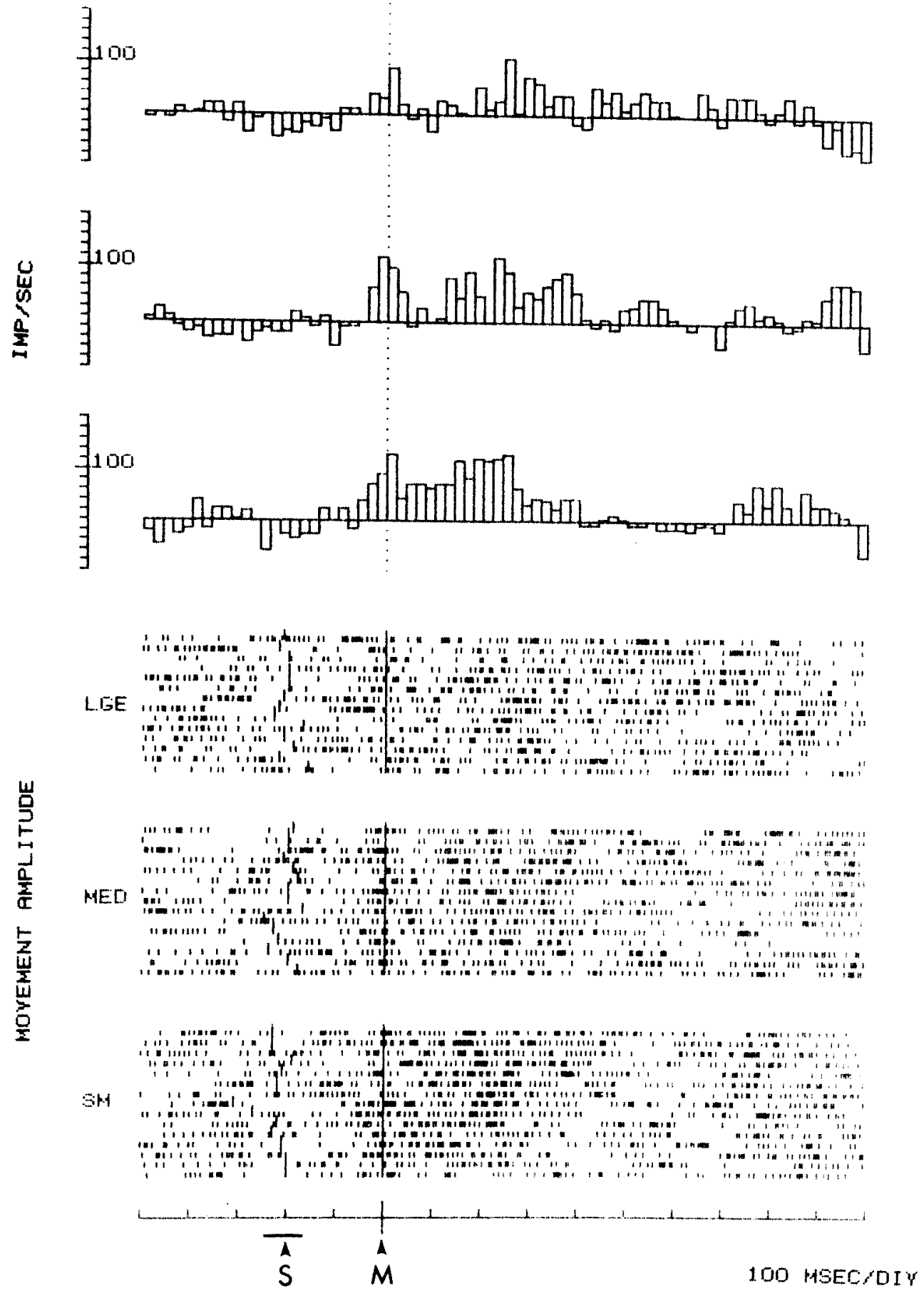

Figure 4. Rasters and histograms (difference from mean control discharge rates) of the activity of an SNpr neuron (HDR type) whose discharge was related to the direction (not shown) and amplitude of movement. $S$, appearance of the target stimulus (horizontal bar $= \pm 1 \mathrm{SD}) ; M$, onset of movement. Binwidth of histograms is $20 \mathrm{msec}$.

(4) the recent observation that intracerebral transplants of embryonic SN neurons, which almost certainly lack their normal afferent inputs, can compensate for behavioral disturbances following nigrostriatal DA pathway lesions (Bjorklund et al., 1981). It is possible that the level of tonic DA release in the striatum may be modulated in the SNpc by graded changes in the firing rate of individual neurons or by recruitment of additional neurons.

The lack of phasic modulation of the activity of neu- rons in the SNpc does not rule out the possibility that DA release in the striatum could be phasically regulated by local mechanisms within the striatum, such as presynaptic modulation of DA terminals by thalamic and cortical afferents (Dray, 1979; Roberts and Anderson, 1979). Chesselet et al. (1983) have recently summarized the evidence that the release of dopamine from terminals of nigrostriatal neurons can be regulated at the presynaptic level by numerous neurotransmitters present in striatal neurons or striatal afferents. Thus, a lack of 


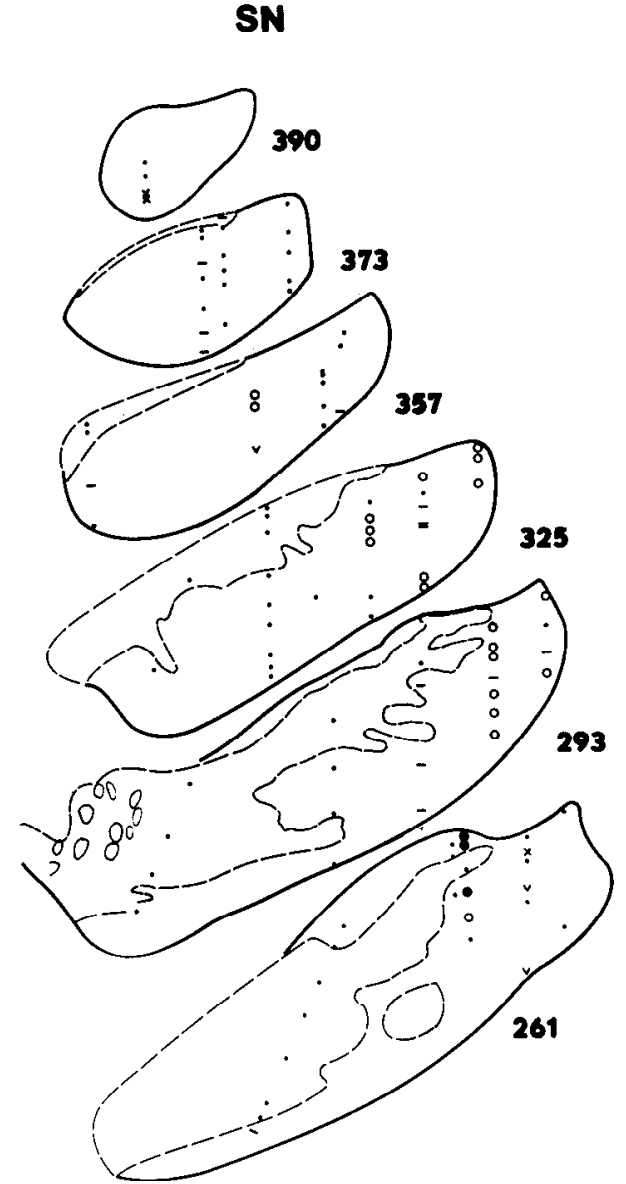

Figure 5. Location of cells of different categories in two animals: Arm, solid circles; orofacial, open circles; axial: $X$; eye movement or visual, $V$; nonspecific, horizontal dashes; nonresponsive, small dots. Higher numbers represent rostral sections.

phasic modulation of SNpc neurons does not necessarily rule out a phasic action of dopamine within the striatum, although it does suggest that such phasic release is independent of impulse flow in the nigrostriatal pathway. It is conceivable that the observed slow tonic discharge of SNpc neurons provides a continuous release of DA in the striatum, while the phasic and possibly more specific actions of DA are regulated at the presynaptic level by other striatal afferents (e.g., corticostriatal or thalamostriatal) or intrinsic neuronal connections.

\section{SNpr}

The finding that a large proportion of neurons in the $\mathrm{SNpr}$ was related to orofacial movements confirms the observation of Mora et al. (1977), who found neurons related to licking and chewing in the general region of the $\mathrm{SN}$ in the behaving monkey. These findings suggest a role of the SNpr in the control of orofacial movements in feeding and licking. It is perhaps relevant clinically that in tardive dyskinesia, which results from long-term treatment with DA receptor blockers, orolingual dyskinesias are the most common symptom (Crane, 1968). Since evidence both for release of DA from the dendriles of SNpc neurons within the SN (Nieoullon, et al., 1977) and for modulation of $\mathrm{SNpr}$ firing rates by iontophoretically applied DA (Ruffieux and Shultz, 1980) has been found, it is conceivable that chronic blockade of DA receptors in the $\mathrm{SN}$ might lead to changes in receptor sensitivity. This, in turn, might result in the involuntary orolingual buccal movements in tardive dyskinesia. The apparent greater proportion of SNpr neurons related to orofacial than to limb movement might account for the prevalence of orolingual movements in this disorder.

The localization of $\mathrm{OF}$ neurons to the centrolateral portions of the SNpr suggests a somatotopic organization of this nucleus, as does the report of weak projections from the face area of the motor cortex to this portion of the SNpr (Kunzle, 1976). The paucity of identified arm or leg neurons in this study is surprising, however, since areas of the putamen which are related to the arm and leg movements are known to project to the SNpr, particularly to its more caudal portions (Szabo, 1967). It is noteworthy in this regard that five of the seven arm neurons in this study were in the caudal portions of the nucleus. Hikosaka and Wurtz (1981) have also found SNpr cells related to saccadic eye movements primarily in the lateral portions of the nucleus.

The high discharge rates and pattern of firing of neurons in the SNpr closely resemble those of neurons in the internal segment of the globus pallidus (GPi). Morphological similarities between $\mathrm{GPi}$ and $\mathrm{SNpr}$ have been repeatedly observed (Mitro, 1896; Olszewski and Baxter, 1954; Fox et al., 1974; Parent et al., 1977). Neurons related to licking and chewing movements are found in both the lateral portion of SNpr and in the ventrocaudal portions of GPi. The grouping of similar neurons on

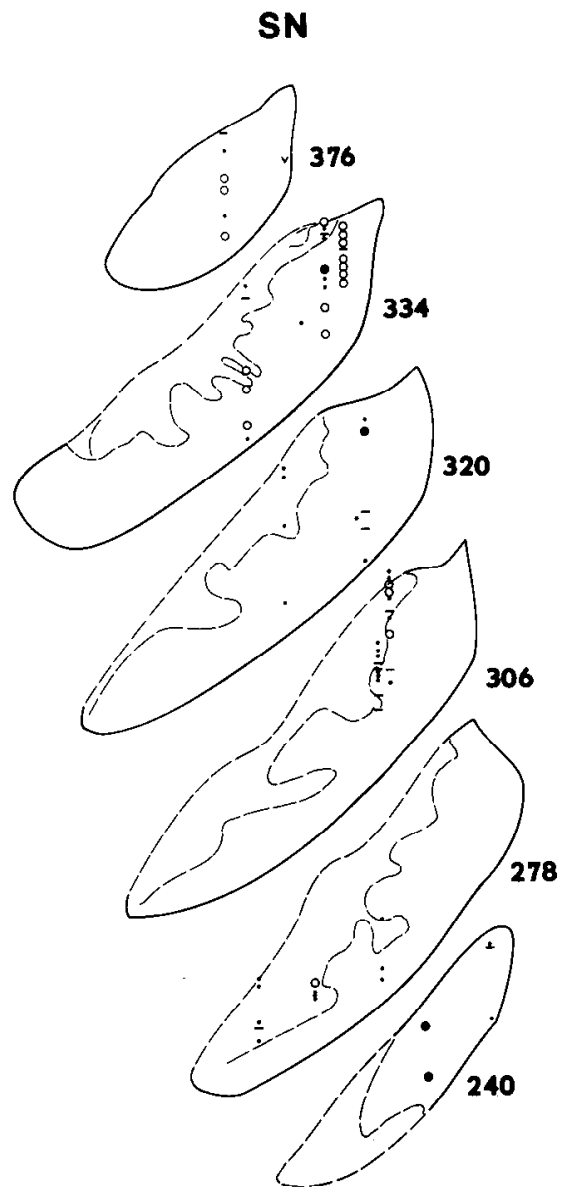

Figure 6. Location of cells of different categories in a third animal. Same conventions as in Figure 5. 
opposite sides of the internal capsule and the similarities in discharge properties of SNpr and GPi neurons, together with the strong morphological and anatomical similarities of GPi and SNpr, led us (DeLong and Georgopoulos, 1979, 1981) to propose that the SNpr and GPi represent medial and lateral portions of a single functional entity which has been divided by the internal capsule. A similar view has been proposed independently by others (Nauta, 1979). This view is given further support by the observation that in certain mammals (whales and porpoises) the internal capsule does not separate the GP from the SN, which are combined in a single structure (Riese, 1924).

\section{References}

Anderson, M. E. (1976) Tonic firing patterns of substantia nigra neurons in awake monkeys. Soc. Neurosci. Abstr. 2: 59.

Bjorklund, A., U. Stenevi, S. B. Dunnett, and S. D. Iverson (1981) Functional reactivation of the deafferented neostriatum by nigral transplants. Nature 289: 497-499.

Bunney, B. S., J. R. Walters, R. H. Roth, and G. Aghajanian (1973) Dopaminergic neurons: Effects of antipsychotic drugs and amphetamine on single cell activity. J. Pharmacol. Exp. Ther. 185: 560-571.

Carpenter, M. B., K. Nakano, and R. Kim (1976) Nigrothalamic projections in the monkey demonstrated by autoradiographic technics. J. Comp. Neurol. 165: 401-416.

Chesselet, M. -F., A. Cheramy, T. Reisine, C. Lubetzki, and J. Glowinski (1983) Presynaptic regulation of striatal dopamine release: In vivo and in vitro studies. J. Physiol. (Paris), in press.

Crane, G. E. (1968) Am. J. Psychiatry 124 (Suppl.): 40.

Crutcher, M. D., and M. R. DeI Ing (1981) Relation of putamen neuronal discharge to direction of movement or pattern of muscular activity. Soc. Neurosci. Abstr. 7: 778.

Crutcher, M. D., and M. R. DeLong (1983) Single cell studies of the primate putamen. II. Relations to direction of movement and pattern of muscular activity. Exp. Brain Res., in press.

DeLong, M. R., and A. P. Georgopoulos (1978) The subthalamic nucleus and the substantia nigra of the monkey. Neuronal activity in relation to movement. Soc. Neurosci. Abstr. 4: 42.

DeLong, M. R., and A. P. Georgopoulos (1979) Motor functions of the basal ganglia as revealed by studies of single cell activity in the behaving primate. In Advances in Neurology, L. J. Poirier, T. L. Sourkes, and P. J. Bedard, eds., Vol. 24, pp. 131-140, Raven Press, New York.

DeLong, M. R., and A. P. Georgopoulos (1981) Motor functions of the basal ganglia. In Handbook of Physiology, Section 1: The Nervous System, Vol. II. Motor Control, Part 1, J. M. Brookhart, V. B. Mountcastle, and S. R. Geiger, eds., pp. 1017-1061, American Physiological Society, Bethesda, MD.

Dray, A. (1979) The striatum and substantia nigra: A commentary on their relationships. Neuroscience 4: 1407-1439.

Fallon, J. H., and R. Y. Moore (1978) Catecholamine innervation of the basal forcbrain. IV. Topography of the dopamine projection to the basal forebrain and neostriatum. J. Comp. Neurol. 180: 545-580.

Feger, J., and C. Ohye (1975) The unitary activity of the substantia nigra following stimulation of the striatum in awake monkey. Brain Res. 89: 155-159.

Feger, J., J. Jacquemin, and C. Ohye (1978) Peripheral excitatory input to substantia nigra. Exp. Neurol. 59: 351-360.

Fox, C. A., N. Andrade, I. J. LuQui, and J. A. Rafols (1974) The primate globus pallidus: A Golgi and electron microscopic study. J. Hirnforsch. 15: 74-93.

Georgopoulos, A. P., M. R. DeLong, and M. D. Crutcher (1983) Relations between parameters of step-tracking movements and single cell discharge in the globus pallidus and subthalamic nucleus of the behaving monkey. J. Neurosci. 3: $1586-1598$

Graybiel, A. M. (1978) Organization of the nigrotectal connection: An experimental tracer study in the cat. Brain Res. 143: 339-348.

Guyenet, P. G., and G. K. Aghajanian (1978) Antidromic identification of dopaminergic and other output neurons of the rat substantia nigra. Brain Res. 150:69-84.

Hikosaka, O., and R. H. Wurtz (1981) The role of the substantia nigra in the initiation of saccadic eye movements. In Progress in Oculomotor Research, A. Fuchs and W. Becker, eds., pp. 145-152, Elsevier, New York.

Hommer, D. W., and B. S. Bunney (1980) Effect of sensory stimuli on the activity of dopaminergic neurons: Involvement of non-dopaminergic nigral neurons and striato-nigral pathways. Life Sci. 27: 377-386.

Jung, R., and R. Hassler (1960) The extrapyramidal motor system. In Handbook of Physiology: Neurophysiology J. Field, H. W. Magoun, and V. E. Hall, eds., Sect. 1, Vol. II, chap. 35, American Physiological Society, Bethesda, MD.

Künzle, H. (1976) Thalamic projections from the precentral motor cortex in Macaca fasciscularis. Brain Res. 105: 253267.

Martin, J. P. (1967) The Basal Ganglia and Posture, Pitman, London.

Mehler, W. R. (1971) Idea of a new anatomy of the thalamus. J. Psychiatr. Res. 8: 203.

Mitro, D. (1896) Contribute alla fina anatomia della substantia nigra di Sommering e del pedunculo cerebralle dell'uomo. Riv. Sper. Freniat. 22: 197-210.

Mora, F., G. F. Mogenson, and E. T. Rolls (1977) Activity of neurons in the region of the substantia nigra during feeding in the monkey. Rrain Res. 1.33: 267-276.

Nauta, H. J. W. (1979) A proposed conceptual reorganization of the basal ganglia and telencephalon. Neuroscience 4: 18751881 .

Nieoullon, A., A. Cheramy, and J. Glowinski (1977) Release of dopamine in vivo from cat substantia nigra. Nature 266: 375 377.

Olszewski, J., and D. Baxter (1954) Cytoarchitecture of the Human Brain Stem, J. B. Lippincott, Philadelphia.

Parent, A., L. J. Poirier, R. Boucher, and L. L. Butcher (1977) Morphological characteristics of acetylcholinesterase containing neurons in the CNS of DFP-treated monkeys. Part 2. Diencephalic and medial telencephalic structures. J. Neurol. Sci. 32: 9-28.

Riese, W. (1924) Zuz vergleichenden anatomie der Striofugalen Faserung. Anat. Anz. 57: 487-494.

Roberts, P. J., and S. D. Anderson (1979) Stimulatory effect of L-glutamate and related amino acids on $(3 /+)$ dopamine release from rat striatum. J. Neurochem. 32: 1539-1545.

Ruffieux, A., and W. Schultz (1980) Dopaminergic activation of reticula neurons in the substantia nigra. Nature 285: $240-$ 241.

Steinfels, G. F., J. Heym, and B. J. Jacobs (1981) Single unit activity of dopamincrgic neurons in frcely moving cats. Life Sci. 29: $1435-1442$.

Stern, G. (1966) The effects of lesions in the substantia nigra. Brain 89: 449-451.

Szabo, J. (1967) The efferent projections of the putamen in the monkey. Exp. Neurol. 19: 463-178.

Ungerstedt, U. (1971) Adipsia and aphasia after 6-hydroxydopamine induced degeneration of the nigro-striatal dopamine system. Acta Physiol. Scand. 82 (Suppl. 367): 92-122.

Viallet, F., E. Trouche, D. Beaubaton, A. Nieoullon, and E. Legallet (1981) Bradykinesia following unilateral lesions restricted to the substantia nigra in the baboon. Neurosci. Lett. 24: $97-102$. 